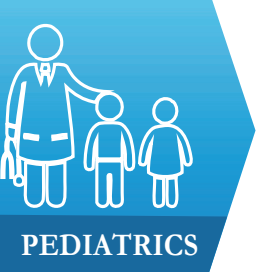

1) Department of Pediatrics I, Iuliu Hațieganu University of Medicine and Pharmacy, Cluj-Napoca, Romania

2) Pediatric Clinic I, Pediatric Emergency Hospital, Cluj-Napoca, Romania

\title{
Bruising as the first sign of exocrine pancreatic insufficiency in infancy
}

Csilla Enikő Szabo ${ }^{1,2}$, Oana Iulia Man², Radu Sorin Șerban ${ }^{1,2}$, Eva Kiss ${ }^{1,2}$, Călin Florin Lazăr ${ }^{1,2}$

\begin{abstract}
Exocrine pancreatic insufficiency is an important cause of chronic malnutrition, secondary to maldigestion-malabsorption, which can be caused in children especially by cystic fibrosis, but also by other much rarer diseases. The case of a 6 months and 3 weeks old male pediatric patient is reported, who was admitted to the clinic for head and forearms bruising. Laboratory findings identified vitamin $\mathrm{K}$ deficiency as the cause of the cutaneous hemorrhagic syndrome. Further investigations revealed association of steatorrhea (which is a marker of fat malabsorption), iron-deficiency anemia and hypovitaminosis $\mathrm{D}$, which had been produced by nutritional deficiencies caused by malabsorption syndrome. From the numerous disorders that could be associated with pancreatic insufficiency in children, the following conditions had been excluded: cystic fibrosis (mucoviscidosis), cow`s milk protein intolerance, gluten-sensitive enteropathy (coeliac disease), Shwachman-Diamond syndrome, abetalipoproteinemia, etc. Based upon decreased levels of stool pancreatic elastase in repeated measurements, together with low serum lipase, the final diagnosis of exocrine pancreatic insufficiency was established. Treatment of this case consisted mainly in pancreatic enzyme replacement therapy, but also oral iron supplementation and dietary supplements with fat-soluble vitamins (A, D, E, K). The outcome was favorable, characterized by normalization of intestinal passage, ascending growth curve and normalization of the majority of laboratory tests values that were modified between the time of patient admission to our clinic and initiation of specific therapy (serum level of vitamin K, vitamin D and lipase, coagulation profile, hemoglobin and red blood cell indexes), as well as higher value of fecal pancreatic elastase.
\end{abstract}

Keywords: bruising, vitamin $\mathrm{K}$ deficiency, steatorrhea, malabsorption, exocrine pancreatic insufficiency

\section{Introduction}

Exocrine pancreatic insufficiency is one of the maldigestion-malabsorption syndromes, thus being associated with poor digestion and, subsequently with diminished intestinal absorption of one or more dietary nutrients. Exocrine pancreatic insufficiency is determined by various pancreatic enzyme deficiencies causing an alteration at the level of the intestinal lumen which results in chronic diarrhea and steatorrhea, that are in turn responsible for the development of multiple nutrient deficiencies, including fats, proteins, carbohydrates, vitamins (especially fat-soluble A, D, E, K) and electrolytes, with specific clinical consequences [1-3]. However, fat maldigestion-malabsorption seems to be the most important physiopathological process, which explains why steatorrhea is the main clinical symptom of exocrine pancreatic insufficiency. Steatorrhea is defined as the presence of excess fat in the stool and it can be manifested by diarrhea, large bulky, oily or greasy stools, increased gas content or stool floating on the toilet water. The most common cause of exocrine pancreatic insufficiency in children is represented by cystic fibrosis, but there are several much rarer possible etiologies, such as the following syndromes: Shawchman-Diamond, Johanson-Blizzard, Pearson or various isolated enzyme deficiencies. Therapeutic strategy in this disease consists first in pancreatic enzyme replacement therapy, in association with dietary management, 
which would ensure a favorable prognosis. On the other hand, patients with untreated exocrine pancreatic insufficiency not only suffer from impaired quality of life due to steatorrhea, weight loss, abdominal discomfort and other symptomes related to this disease, but are also at high risk to develop nutritional deficiencies with specific secondary disorders, such as: hemeralopia, xerophtalmia, keratosis follicularis (vitamin A deficiency), bone decalcification with rickets or osteomalacia (vitamin D deficiency), hematologic disorders - anemia, neurological, neuromuscular and eye and visual system disorders (vitamin E deficiency), hemorrhagic syndrome, colitis, diarrhea, steatorrhea (vitamin K deficiency) etc [1,4-6]. Therefore, it is essential to diagnose and treat this pancreatic disorder properly, in order to prevent possible complications that could occur in time and have an obviously negative impact on the patient's life quality.

A case of exocrine pancreatic insufficiency is reported, in which the diagnosis was established after an initial episode of cutaneous hemorrhagic syndrome caused by vitamin K deficiency. Subsequently, steatorrhea (both being consequences of fat malabsorption), iron-deficiency anemia and hypovitaminosis $\mathrm{D}$, as nutritional deficiencies produced by malabsorption syndrome, had been identified.

\section{Case report}

The patient reported here is a male infant, without any known allergies, coming from organized, wealthy, quite young and healthy parents, living in the urban area. He was admitted to the Pediatric Clinic I from Pediatric Emergency Hospital Cluj-Napoca at the age of 6 months and 3 weeks for insidious onset bruises located on the head and forearms. Early personal history of the patient revealed that he was born after a full term and uneventful pregnancy to a $\mathrm{G} 2 \mathrm{P} 2$ mother, via vaginal delivery, with normal weight and length at birth, he had physiological neonatal jaundice, and there were no postnatal complications. He was breastfed for 6 months and then started eating solid foods. Before admission to the clinic, prophylaxis of rickets had been made correctly (1000 units of vitamin D per day) and he had been vaccinated according to national standards.

Clinical examination of the patient at the first presentation revealed a good general condition, but growth delay of $-22.02 \%$ (weighted index $=0.83$; nutritional index $=0.77$ ), reduced subcutaneous adipose tissue, skin and mucous pallor, a bruise of approximately $5 / 5 \mathrm{~cm}$ in the parietal region of the head and multiple bruises with diameters between 1 and $4 \mathrm{~cm}$ on the forearms, bloating, minor bone deformities of the thorax (rosary, increased antero-posterior diameter of the chest wall) and head (frontal and parietal skull bossing), grade I/II systolic murmur in left parasternal area, without any other pathological findings at the examined organ systems (Figure 1). Based upon the patient's history and clinical findings, the following diagnosis was established: cutaneous hemorrhagic syndrome, anemic syndrome, rickets signs, grade I/II systolic murmur, grade 1 protein-calorie malnutrition.

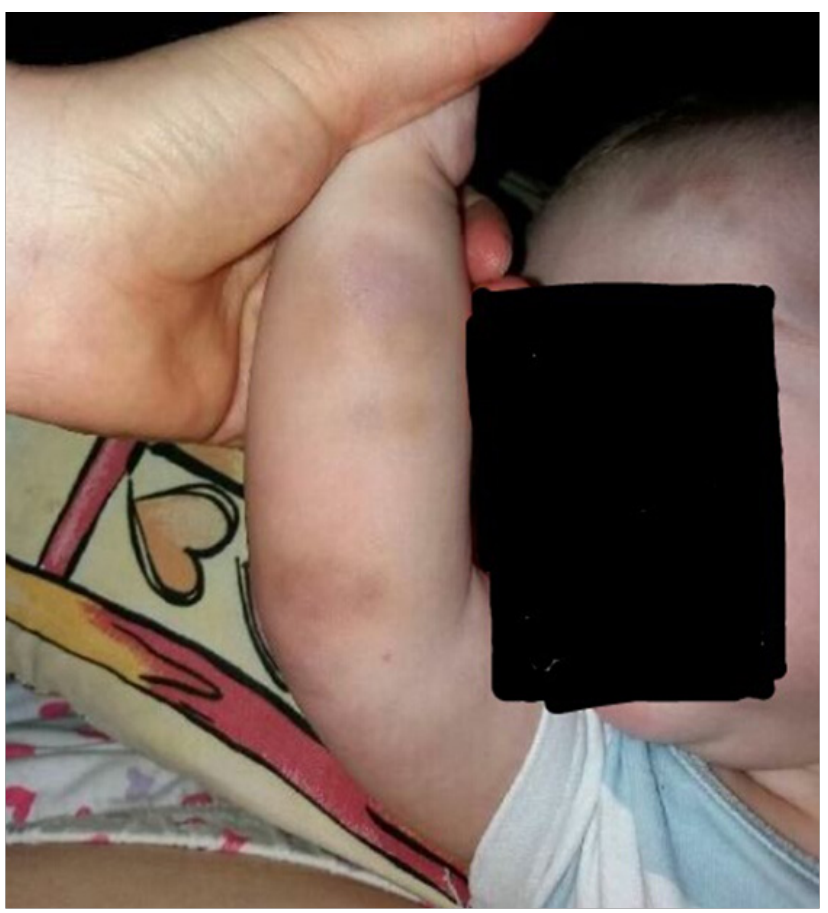

Figure 1. Physical findings at the first presentation - head and forearms bruising

Disorders of hemostasis can be classified into vascular, platelet and coagulation factors defects. Each one of the previously mentioned anomalies can be hereditary or acquired $[1-3,7]$.

Laboratory findings in this stage revealed: prothrombin time 75\% (normal range $80-120 \%$ ), serum fibrinogen $128 \mathrm{mg} / \mathrm{dl}$ (normal range 180-450 mg/dl), thrombin time $23.9 \mathrm{sec}$ (normal range 14-21 sec), serum vitamin $\mathrm{K}-\mathrm{K} 1=0.15 \mu \mathrm{g} / 1$ (normal range $0.2-3 \mu \mathrm{g} / \mathrm{l}$ ) and $\mathrm{K} 2$ : MK-4 $=0.23 \mu \mathrm{g} / \mathrm{l}$, MK-7 $=0.1 \mu \mathrm{g} / \mathrm{l}$. Complete blood count was suggestive for normochrome, normocytic anemia: hemoglobin $9.9 \mathrm{~g} / \mathrm{dl}$ (normal range 11-14.5 g/dl), hematocrit $30.1 \%$ (normal range $33-42 \%$ ), red cell count $4,170,000 / \mu \mathrm{l}$ (normal range 4,000,000-5,300,000/ $\mu \mathrm{l}$ ), $\mathrm{MCV}$ $72.2 \mathrm{fL}$ (normal range 72-89 fL), MCH $23.7 \mathrm{pg}$ (normal range 25-31 pg), MCHC $32.9 \mathrm{~g} / \mathrm{dl}$ (normal range 32-37 g/ dl). Biochemical laboratory tests showed low blood level of ferritin: $10 \mathrm{ng} / \mathrm{ml}$ (normal range 12-327 ng/ml), which could represent the cause of iron deficiency anemia. Stool sample analysis identified high fat content, which is a marker of lipid malabsorption from ingested food.

Malabsorption can be primary or secondary to maldigestion and it can be in its turn either global or selective - meaning malabsorption of lipids or carbohydrates or proteins. The possible causes of fat malabsorption can be grouped in hepatobiliary disorders (such as hepatitis, 
cirrhosis, cholestatic syndromes etc.), pancreatic and intestinal diseases, respectively [1-3].

At the next admission of the patient to the clinic, additional paraclinical investigations were performed, in order to establish the etiology of the malabsorption syndrome and also to follow up the patient's condition. Paraclinical assessment revealed normal coagulation profile after the oral administration of vitamin K. Furthermore, anemia was corrected after oral iron supplementation for 3 months. Peripheral blood smear was then examined and as a result, the following possible etiologies of the malabsorption syndrome could be excluded: ShwachmanDiamond syndrome (which is characterized by neutropenia) and abetalipoproteinemia (which is associated with acantocytosis) [1]. Liver enzymes (alanine and aspartate aminotransferases, alkaline phosphatase, gammaglutamyltransferase), as well as direct and total bilirubin were within normal limits, so a possible hepatobiliary disorder could be excluded as a cause of fat malabsorption syndrome. Serum lipid profile did not reveal any disorders of blood lipids; on the other hand, dosing of serum pancreatic enzymes showed lipase deficiency: $67 \mathrm{U} / 1$ (normal range 73-393 U/1), but normal serum amylase level: $89 \mathrm{U} / 1$ (normal range 30-100 U/1). Immunological investigations excluded cow's milk protein intolerance (specific IgE to beta-lactoglobulin $\mathrm{f} 77$ level was less than $0.100 \mathrm{kU} / \mathrm{l}$ ) and gluten-sensitive enteropathy (both tissue transglutaminase and endomysial IgA antibodies were negative) from the list of intestinal disorders possibly involved in malabsorption syndrome. Laboratory tests also revealed hypovitaminosis D: $12.5 \mathrm{ng} / \mathrm{ml}$ (normal range 30-100 ng/ml) and selective immunoglubulin $\mathrm{G}$ and $\mathrm{M}$ deficiency. Stool sample analysis was negative for Giardia infection. Fecal pancreatic elastase level was less than $15 \mu \mathrm{g} / \mathrm{g}$ (normal values $>200 \mu \mathrm{g} / \mathrm{g}$ ) in repeated examinations, that confirmed exocrine pancreatic insufficiency. Abdominal ultrasonography showed normal structural aspect of the pancreas, as well as of the liver and intestine. Given that cystic fibrosis is the most common cause of exocrine pancreatic insufficiency in children and there were some anamnestic and clinical criteria to take this disease into consideration as a possible diagnosis in this case, a chloride sweat test was performed using quantitative pilocarpine iontophoresis (through Wescore method, by Nanoduct device). It showed a concentration of $20 \mathrm{mmol} / \mathrm{l}$ sodium chloride and $8 \mathrm{~g} / \mathrm{m}^{2} / \mathrm{min}$ amount of sweat, that meant negative test or unlikely Mucoviscidosis diagnosis. According to the Therapeutic Protocol for Cystic Fibrosis (Mucoviscidosis), whenever clinical presentation is suggestive for cystic fibrosis, but the result of sweat chloride test is negative or inconclusive, molecular genetic analysis of the 34 most frequent CFTR gene mutations is mandatory [8]. Genetic analysis only revealed a benign polymorphism without phenotypic expression of the disease, thus, cystic fibrosis could be excluded.

Based upon the clinical and paraclinical data described above, the final diagnosis of exocrine pancreatic insufficiency was established. It caused vitamin $\mathrm{K}$ and D deficiency, as well as iron deficiency anemia (iron absorption disorder was most probably secondary to the pancreatic insufficiency).

Treatment of this patient mainly consisted in pancreatic enzyme replacement therapy (with Pancreatinum 10,000 units using an initial dose of 2500 units/meal and then 3000 units/meal, because steatorrhea reappeared and growth curve for weight and length was abnormal), in association with dietary supplements containing fatsoluble vitamins (A, D, E, K) and oral iron supplements for the treatment of iron-deficiency anemia, in a dose of 5 $\mathrm{mg} / \mathrm{kg} /$ day for 3 months. On this treatment, the patient had a favorable outcome, sustained at the presentation in the clinic after 1 year and 2 months from the first admission (at the age of 1 year and 8 months), by the clinical results (ascending growth curve for weight and length, normalization of intestinal passage, disappearance of bruising), as well as by the paraclinical tests (normalization of coagulation, complete blood count and biochemistry profile values). However, at that moment, a slightly growth delay of $-12.89 \%$ persisted, with $10 \mathrm{~kg}$ weight and $82 \mathrm{~cm}$ height (-0.42 SD), the weighted index $=0.89$, the nutritional index $=0.87$ and the $Z$ score $=-1.15$. The patient had normally colored skin, without pallor or bruises, atopic dermatitis, persistent vitamin D dependent rickets signs consisting in head and thorax bone deformities. Paraclinical assessment showed: normalization of hemoglobin and red blood cell indexes, normal level of vitamin $\mathrm{D}$, selective deficit of $\operatorname{IgG}$ ( $\operatorname{IgA}$ and $\operatorname{IgM}$ values were normal), normal serum lipase level (89 U/1), low concentration of pancreatic elastase in feces but higher than the value at the admission to the clinic $(60 \mathrm{ug} / \mathrm{g})$. Stool sample analysis revealed neither fat, nor markers of infection or bleeding. The sweat chloride test was also repeated and it showed normal value of sodium chloride $(21 \mathrm{mmol} / \mathrm{l})$ and a higher amount (7 $\mathrm{g} / \mathrm{m}^{2} / \mathrm{min}$ ) of sweat, that meant inconclusive test result. Abdominal ultrasound did not describe any pathological aspects. In consequence, at the patient's discharge, it was recommended to continue pancreatic enzymes replacement therapy and fat-soluble dietary supplements.

\section{Discussion}

Normal digestion requires: a. adequate stimulation of pancreatic secretion; $b$. sufficient production of digestive enzymes by pancreatic acinar cells and physiological action of amylase, trypsin, chymotrypsin and lipase, that are released into the duodenum as a response to the presence in the intestinal lumen of fatty acids, peptides and aminoacids; bicarbonate neutralizes acidity of gastric juice and creates optimum $\mathrm{pH}$ for appropriate functioning of pancreatic enzymes; c. a pancreatic duct system without significant outflow obstruction and $d$. adequate mixing of the pancreatic juice with ingested food. Disorders in 
any of these sequences may result in pancreatic exocrine insufficiency, that is an important cause of maldigestionmalabsorption (mainly of lipids) and leads to steatorrhea, weight-loss and malnutrition-related complications $[1,5]$. Vitamin $\mathrm{K}$ is a fat-soluble vitamin involved in the hemostasis process, more precisely in the coagulation phase. This vitamin is necessary for the synthesis of coagulation factors II, VII, IX and X, as well as of coagulation antagonists represented by $\mathrm{C}, \mathrm{S}$ and $\mathrm{Z}$ proteins, that provide a hemostatic balance, in this way avoiding bleeding or, on the other hand, thrombus formation. Beside S protein (which can be also synthetized in several other tissues), the other proteins are synthetized and activated in the liver, as a result of glutamic acid carboxylation, vitamin $\mathrm{K}$ playing a cofactor role in this process. Therefore, decreased serum level of vitamin K may produce probably the most common acquired bleeding disorder of childhood that can result in any degree of bleeding from minor bleeding to severe, clinically significant, life-threatening hemorrhage. It can cause prolonged prothrombin time, revealed by paraclinical investigations $[1,7,8]$. In this clinical case, steatorrhea might have been present since the onset of the disease, but the cutaneous hemorrhagic syndrome was the revealing sign that suggested the final diagnosis.

There are numerous conditions that may be involved in the etiology of exocrine pancreatic insufficiency and that is the reason why the differential diagnosis of this disease should be made with the following disorders, presented in decreasing range of frequency: a. Cystic Fibrosis (the most common lethal genetic disease, caused by autosomal-recessive CFTR$7 \mathrm{q} 31$ gene mutation and typically characterised by the clinical triad of exocrine pancreatic insufficiency, chronic pulmonary disease and elevated concentration of chloride and sodium in the sweat); b. Shwachman-Diamond syndrome (secondary to an autosomal-recessive mutation in the SBDS gene located on chromosome 7, that produces exocrine pancreatic insufficiency, neutropenia, metaphyseal dysostosis, short stature, weight loss); c. Pearson syndrome (produced by a mutation in the mitochondrial DNA, that leads to exocrine pancreatic insufficiency associated with poor growth and severe bone marrow failure causing severe macrocytic anemia and variable degree trombocytopenia); d. JohansonBlizzard syndrome (caused by a mutation in the UBR1 gene located on chromosome 15 , which is clinically characterized by various signs and symptoms such as: exocrine pancreatic insufficiency, hypoplasia or aplasia of the alae nasi, congenital deafness, hypothyroidism, developmental delay, short stature, ectodermal scalp defects, absence of permanent teeth, urogenital malformations and imperforate anus); e. isolated enzyme deficiencies (trypsinogen, enterokinase, lipase, colipase, amylase) and f. other syndromes associated with pancreatic insufficiency (pancreatic agenesis, congenital pancreatic hypoplasia, congenital rubella, duodenal atresia and stenosis, familial or nonfamilial hyperinsulinemic hypoglicemia that often requires pancreatectomy to be controlled, coeliac disease, malnutrition).

Is is important to mention that the pancreatic function can be measured by direct (stimulatory) and indirect (nonstimulatory) methods, which are other possible investigations for diagnosing disorders of the exocrine pancreas: pancreatic stimulation test using Dreiling triplelumen tube or endoscopic techniques after stimulation with secretin and/or cholecystokinin; fat (microscopic evaluation of fecal samples, 72 hour stool collection for quantitative analysis of fat content, coefficient of fat absorption in relation to fat intake using the modified van de Kamer method of fat extraction), fecal elastase-1 and chymotrypsin in stool, nutritional markers (fat-soluble vitamins, apolipoproteins, total cholesterol, magnesium, retinol-binding protein, calcium, zinc, selenium and carotene), immunoreactive trypsinogen, lipase and amylase in serum, ${ }^{13} \mathrm{C}$-mixed triglyceride breath test, pancreolauryl test [1-6].

Regarding the treatment of pancreatic insufficiency, the most important concept is pancreatic enzyme replacement therapy, that has 3 main goals: to eliminate maldigestion-malabsorption, to alleviate pancreatic exocrine insufficiency-related symptoms and to prevent malnutrition-related morbidity and mortality and disease progression. Currently, there are several Food and Drug Administration approved pancreatic enzyme preparations and replacement therapies (Creon, Zenpep, Pancreaze, Ultresa, Viokase, Pertzye), that should be administered at the beginning of a meal and then one should consider adding extra enzymes during or towards the end of the meal depending on the amount of fat in the diet, in order to mimic the action of endogenous pancreatic enzymes, which are secreted throughout a meal. In addition, the dosage depends on age and weight of the patient: 20004000 units lipase $/ 120 \mathrm{~mL}$ breast milk or formula (infant up to 12 months), 1000 units lipase $/ \mathrm{kg} / \mathrm{meal}$ initially, then titrate per response (12 months - 4 years) and, respectively, 500 units lipase $/ \mathrm{kg} / \mathrm{meal}$ initially, up to maximum of 2500 units lipase $/ \mathrm{kg} / \mathrm{meal}$ or 10,000 units lipase $/ \mathrm{kg} /$ day or 4000 units lipase/g fat ingested per day (children older than 4 years and adults), plus one half the standard meal dose to be given with snacks. A positive response to pancreatic enzyme replacement would be both a significant diagnostic criteria for exocrine pancreatic insufficiency and an efficient therapeutic tool. On the contrary, if there is poor or even lacking response to pancreatic enzyme replacement therapy, one of the following treatment strategies should be taken into account: increase dosing, check compliance with the patient, add acid inhibitor, consider adding enzymes during and towards end of meal, consider microspheres, possibly adding a rapid release enzyme preparation, look for evidence of concurrent gastrointestinal comorbidities such as: lactose intolerance, enteric bacterial infection, parasites (especially Giardia), small intestinal bacterial overgrowth, biliary disease (cholestasis), colitis, coeliac disease, short bowel syndrome and Chron`s disease. In 
addition, associated to the medical treatment discussed above, lifestyle management is also important, by using hypercaloric diet with high meal frequency, but not increased meal size and at the same time by adding food supplements with fat-soluble vitamins A, D, E, K. However, it is not obvious whether reducing fat intake is beneficial in this disease or not, because results of different research studies are currently contradictory [1,4-6].

The particularity of the reported case is represented by the absence of any clinical specific signs or symptoms of the cutaneous hemorrhagic syndrome caused by vitamin $\mathrm{K}$ deficiency before the age of 6 months, that caused later established diagnosis. Vitamin $\mathrm{K}$ comprises a group of 10 structurally similar, fat-soluble compounds, including 3 main synthetic forms: a. vitamin K1 (phylloquinone, phytomenadione), produced by plants and found in high amounts in green leafy vegetables; b. vitamin K2 (menaquinone) which is synthetised by bacteria in the gut flora, but is also found in animal products; c. vitamin K3 (menadione), which is represented by several water-soluble analogues, which are used for therapeutical purposes. There are 2 etiological forms of vitamin $\mathrm{K}$ deficiency caused bleeding: idiopathic and secondary to various additional factors, which impair the normal vitamin K supply, such as: poor intake, reduced absorption or increased pathological consumption of vitamin K. Furthermore, vitamin K deficiency bleeding can be classified by age at onset into 3 categories: early (within the first 24 hours), classic (days 2 to 7 - mostly 3 to 5) and late (week 2 to 6 months - mostly weeks 2 to 8 ), the last form being the rarest one. Bleeding due to vitamin $\mathrm{K}$ deficiency as a result of fat malabsorption can occur in children of any age and it can be prevented by using oral vitamin $\mathrm{K}$ food supplements (2-3 mg/day) or parenteral intravenous vitamin $\mathrm{K}(1-2 \mathrm{mg} /$ dose). On the other hand, if the clinical signs and symptoms of vitamin $\mathrm{K}$ deficiency as a consequence of malabsorption are already present, patients require prolonged administration of high doses of oral vitamin $\mathrm{K}$ ( $2.5 \mathrm{mg}$ twice/week to $5 \mathrm{mg}$ /day) or parenteral vitamin $\mathrm{K}$ (if oral intake is ineffective) for the treatment of this disease. It is possible to adjust the dose depending on prothrombin time, which is closely monitored $[1,7,9]$.

Another particular feature of the case is the relatively atypical onset of the exocrine pancreatic insufficiency, with cutaneous hemorrhagic syndrome caused by fat-soluble vitamin K malabsorption. Moreover, pancreatic lipase deficiency in this patient is most likely congenital, so pancreatic enzyme replacement therapy should be maintained throughout his entire lifetime, but with the possibility to titrate the dosage based upon clinical manifestations (disappearance or persistence of steatorrhea, the aspect of the growth curve for weight and height).

\section{Conclusion}

The present case report provides evidence that pancreatic exocrine insufficiency can be determined by lipase deficiency (either isolated or associated with other enzyme deficiencies) and it may have an atypical onset, directly with bruising as a sign of fat-soluble vitamin $\mathrm{K}$ deficiency, caused by reduced absorption in the intestine. This case emphasizes the importance of differential diagnosis of cutaneous hemorrhagic syndrome, as well as of malabsorption syndrome. It also highlights the need for early and accurate diagnosis of any exocrine pancreatic disorder and, subsequently, for early and proper treatment, in order to prevent potential complications that could occur in time due to chronic malnutrition.

\section{References}

1. Kliegman RM, Stanton BF, St Geme JW, Schor NF. Nelson Textbook of Pediatrics. 20th ed. Elsevier: Philadelphia; 2016: pp 342-343,1831-1850,1909-1913, 2379-2384, 2397.

2. Grigorescu-Sido P. Tratat elementar de pediatrie. [Essential treatise of pediatrics] vols. 2 and 3. Editura Casa Cărții de Știință: Cluj-Napoca; 1995: pp 386-416, 140-186, 369-385.

3. Ciofu C, Ciofu EP. Esențialul în pediatrie [Essential pediatrics]. 4th edition, Editura Medicală Amaltea: Bucharest; 2017: pp 57, 112-115, 393-400, 407-414, 466-483.

4. Taylor CJ, Chen K, Horvath K, Hughes D, Lowe ME, Mehta D, et al. ESPGHAN and NASPGHAN report on the assessment of exocrine pancreatic function and pancreatitis in children. J Pediatr Gastroenterol Nutr. 2015;61:144-153.

5. Lindkvist B. Diagnosis and treatment of pancreatic exocrine insufficiency. World J Gastroenterol. 2013;19:7258-7266.

6. Struyvenberg MR, Martin CR, Freedman SD. Practical guide to exocrine pancreatic insufficiency - Breaking the myths. BMC Med. 2017; 15:29.

7. Khair K, Liesner R. Bruising and bleeding in infants and children-a practical approach. Br J Haematol. 2006;133:221-231. 8. Pop Liviu Laurenţiu, MD, Professor, Ciucă Ioana Mihaiela, MD, Lecturer. Therapeutic Protocol for Cystic Fibrosis (mucoviscidosis). Timișoara National Center of Mucoviscidosis 2017 September 15.

9. Sutor AH. Vitamin K deficiency bleeding in infants and children. Semin Thromb Hemost.1995;21:317-329. 